\title{
O Estatuto Científico da Epidemilogia
}

\section{The scientific statute of epidemiology}

Se é que a metáfora posicional das assembléias da Revolução Francesa ainda tem alguma valia, a Epidemiologia encontra-se atualmente, na feliz expressão de Jaime Breilh', sob o fogo cruzado da "epidemiologia social", pela esquerda, e da "epidemiologia clínica", pela direita. Por um lado, observa-se uma proposta de constituição de uma "nova" epidemiologia a partir de uma perspectiva hipercrítica (Laurell", 1985), que aparentemente aponta para a negação de todo um processo histórico de organização de um campo de conhecimento de reconhecida eficácia técnica. Por outro lado, orquestra-se um poderoso movimento ideológico de questionamento da validade científica de qualquer investigação epidemiológica que não se apresente claramente como projeto de conhecimento fundado nos sujeitos individuais ${ }^{2.8}$. Nessa situação, a questão da cientificidade da epidemiologia (sem adjetivos) mostra-se crucial para o processo de maturação histórica e institucional da disciplina, com a reavaliação (ou des-construção) das suas bases conceituais e metodológicas.

A primeira geração de epidemiologistas parecia não ter dúvidas sobre o estatuto científico da nova disciplina. Wade Hampton Frost ${ }^{5}$, primeiro professor de epidemiologia da Universidade Johns Hopkins, escreveu em 1927 que "a epidemiologia é essencialmente uma ciência indutiva, preocupada não meramente em descrever a distribuição de doenças, porém sobretudo em compreendê-la a partir de uma filosofia consistente". Outros autores desse período, como Stallybrass ${ }^{21}$, autor do primeiro livro-texto de epidemiologia, e Major Greenwood ${ }^{7}$, primeiro professor de epidemiologia da Universidade de Londres, também reconheciam claramente a epidemiologia como uma ciência.

Dentro do campo de saúde pública, anota-se duas iniciativas sistemáticas no sentido de estabelecer o estatuto científico da epidemiologia. A primeira ocorreu em 1942, em resposta a um editorial do American Journal of Public Health ${ }^{25}$, e o segundo, em 1962, sob a forma de um debate que se seguiu à publicação de um texto-chave de Milton Terris ${ }^{23}$, naquela revista. De acordo com Lilienfeld ${ }^{12}$, um dos poucos elementos de consenso alcançados em ambas oportunidades era que "a epidemiologia é uma disciplina científica".

O manual metodológico pioneiro do campo epidemiológico, escrito por MacMahon e col. ${ }^{13}$, no final da década de 50, abre com a seguinte afirmativa: "epidemiologia é largamente reconhecida como a ciência básica da medicina preventiva". Esta noção de que a Epidemiologia se constitui em ciência básica para a saúde pública, medicina preventiva ou medicina comunitária, já havia sido proposta anteriormente por Morris, no clássico mapeamento do campo epidemiológico denominado "Os Usos da Epidemiologia" ${ }^{16}$. Até hoje Terris ${ }^{24}$, insigne representante do pensamento sanitarista tradicional, defende a posição de que a epidemiologia é a ciência fundamental da saúde pública, verdadeira base técnica do planejamento, administração e implementação de medidas preventivas.

Durante as décadas de 60 e 70 , em paralelo a um notável desenvolvimento de técnicas de coleta e análise de dados epidemiológicos ${ }^{23}$, o debate conceitual sobre a cientificidade da disciplina foi virtualmente reprimido. A idéia de que a epidemiologia é uma variante da "ecologia médica" ou apenas um ramo da ecologia humana será talvez a única contribuição teórica dessa fase. A posição de que a epidemiologia se contitui em um "segmento de uma ciência mais geral" (Stallones ${ }^{20}, 1971$ ), ou ainda que se trata essencialmente de uma disciplina empírica sem maiores demandas teóricas, resultou na crença de que a epidemiologia não é uma ciência.

Lilienfeld"' propôs que seria melhor tomar a epidemiologia como uma "abordagem conceitual" um "modo de pensar" e um "método de raciocínio". Fox e col. ${ }^{+}$afirmam que, pelo fato de não se constituir em um corpo de conhecimentos (que se pode interpretar como não possuir um objeto particular), "a epidemiologia tem sido considerada um método e não uma ciência independente". Para Kleinbaum e col. ${ }^{10}$, a epidemiologia é simultaneamente "uma síntese de conhecimentos oriundos de diversas disciplinas da saúde", "um ramo aplicado da ciência" e uma "disciplina" (no sentido de uma "metodologia" aplicável a qualquer pesquisa em saúde).

Tais afirmações contraditórias e quase incoerentes são sintomáticas da confusão epistemológica predominante nesse campo, derivada da estagnação (ou mesmo retrocesso) observada no desenvolvimento conceitual da epidemiologia moderna. A proposta de uma certa "epidemiologia clínica"3.8.9,18 começou a infiltrar-se por essa brecha, as vezes defendendo o uso da epidemiologia como uma mera fer- 
ramenta para a pesquisa clínica, para logo reduzir, a disciplina ao status mais baixo de uma "abordagem empírica", "uma técnica de resolução de problemas"19.

Mais recentemente, esboça-se uma crescente resistência a tal processo de depreciação. Rothman ${ }^{17}$ concede que a Epidemiologia é um "embrião" entre as ciências (o que, apesar de não parecer, denota uma perspectiva promissora). Susser ${ }^{22}$, por outro lado, tem reconhecido o caráter particular da epidemiologia como uma ciência populacional, que se baseia "nas ciências sociais para uma compreensão da estrutura e da dinâmica sociais (...), na matemática para noções estatísticas de probabilidade, inferência e estimação (...), e sobre as ciências biológicas para o conhecimento do substrato orgânico humano onde as manifestações observadas acharão expressão individual".

Miettinen ${ }^{14}$ registrou na primeira versão (1981) do seu controverso livro que a epidemiologia poderia ser definida como "a ciência da ocorrência de doença". Entretanto, esse mesmo autor posteriormente afirmou que "a epidemiologia não é coerente como uma ciência mas apenas como (...) um agregado de princípios para o estudo da ocorrência de doença e estados ou eventos correlatos"15. O importante é que, para situar-se dessa maneira, Miettinen emprega como método a rigorosa identificação do objeto de conhecimento da epidemiologia, questão fundamental para levantar o problema da cientificidade da disciplina. O mesmo método, porém alcançando conclusão oposta, encontra-se em Goldberg6, que escreveu: "o verdadeiro problema aqui posto (...) é a definição do objeto de estudo. A especificidade de uma ciência é antes de tudo devida ao seu objeto".

Esta é portanto a questão mais fundamental da epidemiologia no presente momento, verdadeiro impasse que poderá, caso não resolvido, implicar uma barreira intransponível para o seu próprio desenvolvimento enquanto campo científico autônomo. Perspectivas epistemológicas mais modernas reconhecem o esgotamento dos modelos formais de delimitação de campos científicos, indicando o papel fundamental dos paradigmas e seus processos históricos, macro e micro sociais, na construção institucional das ciências através da prática, técnica e teoria. Nesse momento crucial, não se deve poupar esforços no sentido da conquista do paradigma, com a construção do objeto epidemiológico tanto pro-positivamente quanto na prática cotidiana de produção de conhecimento. A demarcação de um campo próprio de aplicação será então uma consequiência histórica (e não meramente lógica) desse processo de maturação de uma disciplina que, desde as suas raízes, reafirma a força dos processos sociais na determinação da saúde coletiva.

\section{Referências Bibliográficas}

1. BREILH, J. La epidemiologia entre fuegos. [Apresentado ao Taller Latino Americano de Medicina Social, Medellin, Colombia, jul., 1987]

2. FEINSTEIN, A. Scientific stardards in epidemiologic studies of the menace of daily life. Science, 242: 1257-63, 1988.

3. FLETCHER, R.H.; FLETCHER, S.W.; WAGNER, E. Clinical epidemiology: the essentials. Baltimore, Williams \& Wilkins, 1982.

4. FOX, J.; HALL, C.; ELVEBACK, L. Epidemiology: man and disease. London, MacMillan, 1970.

5. FROST, W.H. Epidemiology. In: Papers of Wade HamptonFrost. New York, Oxford University Press, 1941. p. 493542.

6. GOLDBERG, M. Cet obscur objet de l'épidémiologie. Sci. soc, et Santé, 1 (3): 55-110, 1982.

7. GREENWOOD, M. Epidemiology: historical and experimental. London, Humphrey Milford, 1932.

8. HORWITZ, R. The experimental paradigm and observational studies of cause-effect relationship in clinical medicine. J. chron. Dis., 40 (1): 91-9, 1987.

9. JENICEK, M. \& CLÉROUX, R. Epidémiologie clinique. Québec, Edisen Ind., 1985.

10. KLEINBAUM, D.; KUPPER, L.; MORGENSTERN, H. Epidemiologic research: principles and quantitative methods. Belmont, California, Lifetime Learning Publications, 1982.

11. LAURELL, A.C. Epidemiologia: proceso coletivo saludenfermedad. Inform. cient. y tecnol., 7: 16-9, 1985.

12. LILIENFELD, D. Definitions of epidemiology, Amer. $J$. Epidem., 107: 87-90, 1978.

13. MacMAHON, B.; PUGH, T.; IPSEN, J. Epidemiologic methods. Boston, Little, Brown \& Co., 1960.

14. MIETTINEN, O. Epidemiology as the discipline medical occurrence research. Helsinki, Institute of Occupational Health, 1981. [Fotocópia]

15. MIETTINEN, O. Theoretical epidemiology. New York, John Wiley \& Sons, 1985.

16. MORRIS, J. The uses of epidemiology. Edinburgh, Churchill Livingston, 1957.

17. ROTHMAN, K. Modern epidemiology. Boston, Little, Brown \& Co., 1986.

18. SACKETT, D.; HAYNES, B.; TUGWELL, P. Clinical epidemiology. Boston, Little, Brown \& Co., 1985.

19. SCHUMAN, S. Practice-based epidemiology: an introduction. New York, Gordon \& Breach, 1986.

20. STALLONES, R. Environment, ecology and epidemiology. Washington, D.C., Pan-American Health Organization, 1971. (PAHO - Scientific Publication, 231).

21. STALLYBRASS, C. The principles of epidemiology and the process of infection. London, Routledge, 1931.

22. SUSSER, M. Epidemiology, health \& society: selected papers. New York, Oxford University Press, 1987. p. 8292.

23. TERRIS, M. The scope and methods of epidemiology. Amer. J. publ. Hlth, 52: 1371-6, 1962.

24. TERRIS, M. New perspectives in epidemiology and public health. In: Congresso Brasileiro de Epidemiologia, 1 ${ }^{\circ}$, Campinas, 1990. Anais. Campinas, 1990. p. 253-63.

25. WHAT and who is an epidemiologist? [Editorial]. Amer. $J$. publ. Hlth, 32: 414-5, 1942.

São Paulo, outubro de 1991. Naomar de Almeida Filho Editor Associado da Revista de Saúde Pública Departamento de Medicina Preventiva Universidade Federal da Bahia 